\title{
Excitation and Wireless Lock-In Measurement of Hip Prosthesis Vibrations for Loosening Detection
}

U. Marschner, ${ }^{1}$ Technische Universität Dresden, Institute for Semiconductors and Microsystems Technology, Helmholtzstr. 10, 01062 Dresden; B. Jettkant, Berufsgenossenschaftliches Universitätsklinikum

Bergmannsheil GmbH, Bochum; W.-J. Fischer'; D. Ruwisch, Ruwisch \& Kollegen GmbH, Berlin; H. Grätz, Fraunhofer Institute for Photonic Microsystems IPMS, Dresden, G. Woldt, Microelectronic Packaging

Dresden GmbH, Dresden, B. Clasbrummel, Ev. Krankenhaus Witten im Diakoniewerk Ruhr (Germany)

\begin{abstract}
An experimental setup for excitation and detection of hip prosthesis loosing is developed and analyzed. Small vibration signals are detected by acceleration sensors fixed in the prosthesis. Lock-in technique is applied to separate sensor signals at low signal-to-noise ratio. Stand-alone measurements were taken at an artificial thigh where an electrodynamic audio shaker was fixed at the knee using an appropriate designed support structure. It could be demonstrated that the measurement setup is suitable to excite and to measure oscillations of a prosthesis which was partially fixed in an artificial femur.
\end{abstract}

\section{Introduction}

The detection of hip prosthesis loosening is important to avoid unnecessary revision operations. At present this is the case for approximatly $10 \%$ of all revision operations. These second order surgeries cause excessive costs (app. 15,000 EURO) and human emotional pressure.

It is known that prosthesis loosening changes its mechanical resonance frequencies [1]-[3]. Li and coworkers [2] deduced from their studies that especially late loosening can be reliably diagnosed by vibration technology, but the technique is not sensitive enough to detect early loosening with minimal or no mechanical stability. Qi et al. [4] conducted numerical modal analysis to obtain the natural frequencies and mode shapes of femoral models under free vibration. They concluded that this tool is able to provide reliable diagnosis when more than one-third of the stem length shows an interface failure to the bone.

Detection of resonance frequencies and phase shift of mechanical systems is an established sensitive measurement method. Telemetry systems for the detection of hip prosthesis loosening by vibration analysis have been presented [5]-[11]. Our intention has been to develop a wireless diagnosic system to detect prosthesis loosening with included preprocessing of the measurements. The system consists of an integrated part with sensors and telemetry and an external part with transmitter/receiver and measurement evaluation software. It enables medical practitioners to evaluate the prosthesis condition.

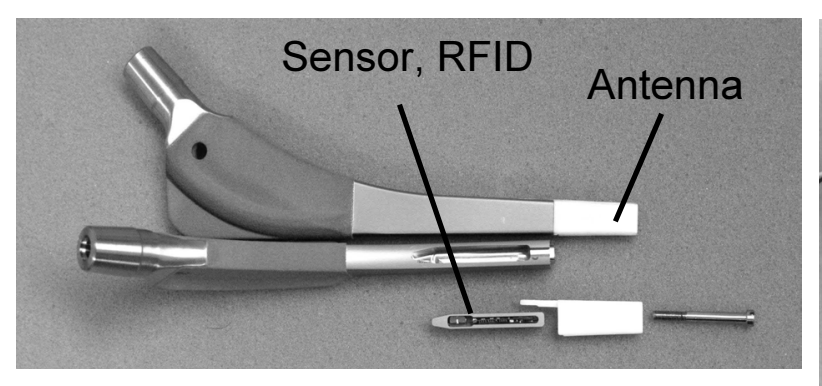
RF field
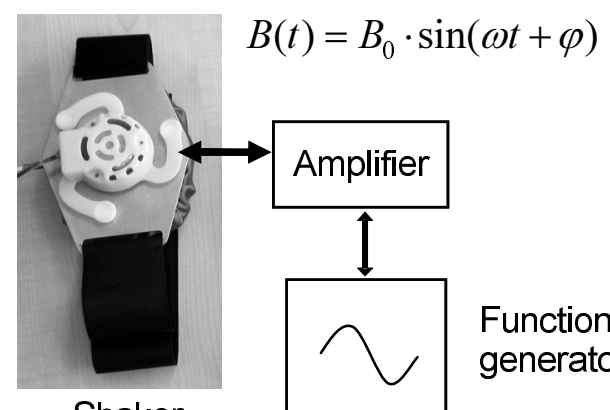

Shaker

Function
$125 \mathrm{kHz}$

generator

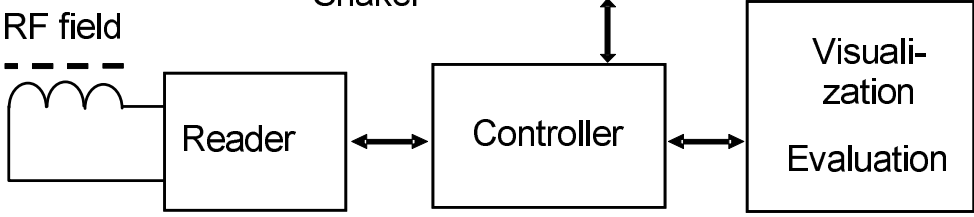

Figure 1: System concept for prosthesis excitation and wireless vibration measurement. The hip prosthesis includes the integrated acceleration sensor, lock-in amplifier and transponder ASIC 
An easy system extension by additional sensors should be achieved since standard interfaces have been implemented. The goal of a medical diagnostics consists also in a reliable acquisition and registration of relevant clinical parameters during longer periods, up to several years.

In the first part of the paper the system construction is decribed followed by the development and investigation of the excitation construction. It was applied to a measurement setup which is presented in the second part of the paper.

\section{System Construction}

Figure 1 depicts the developed system concept for prosthesis excitation and wireless vibration measurement. The shown hip prosthesis includes the integrated acceleration sensor, lock-in amplifier and transponder ASIC. A RFID telemetry chip was designed working at $125 \mathrm{kHz}$. The integrated 16 bit microcontroller is arbitrary programmable and is used for signal processing and control of external sensors, which are connected to the included A/D converter, as well as measurement synchronization. For energy and data transmission a single coil is used. The presented wireless measurement system can be in general applied to vibrational diagnostics [10].

As substantial component in the transponder module a Lock-in amplifier was developed in cooperation with the Fraunhofer IPMS. It separates the measurement signal from the noise which is a problem of available miniaturized acceleration sensors. The employment of the lock-in amplifier requires the supply of a synchronisation signal with excitation frequency and certain phase beside the excitation signal. This signal is generated by the transponder, which receives the necessary information from the reader as shown in Figure 2. For the external generation of the signal an integrated sinus-wave generator was programmed.

Special attention had to be paid to the excitation of the prosthesis vibrations which are ideally reproducable during a patients examination. The development and characterization of an excitation system is described next.

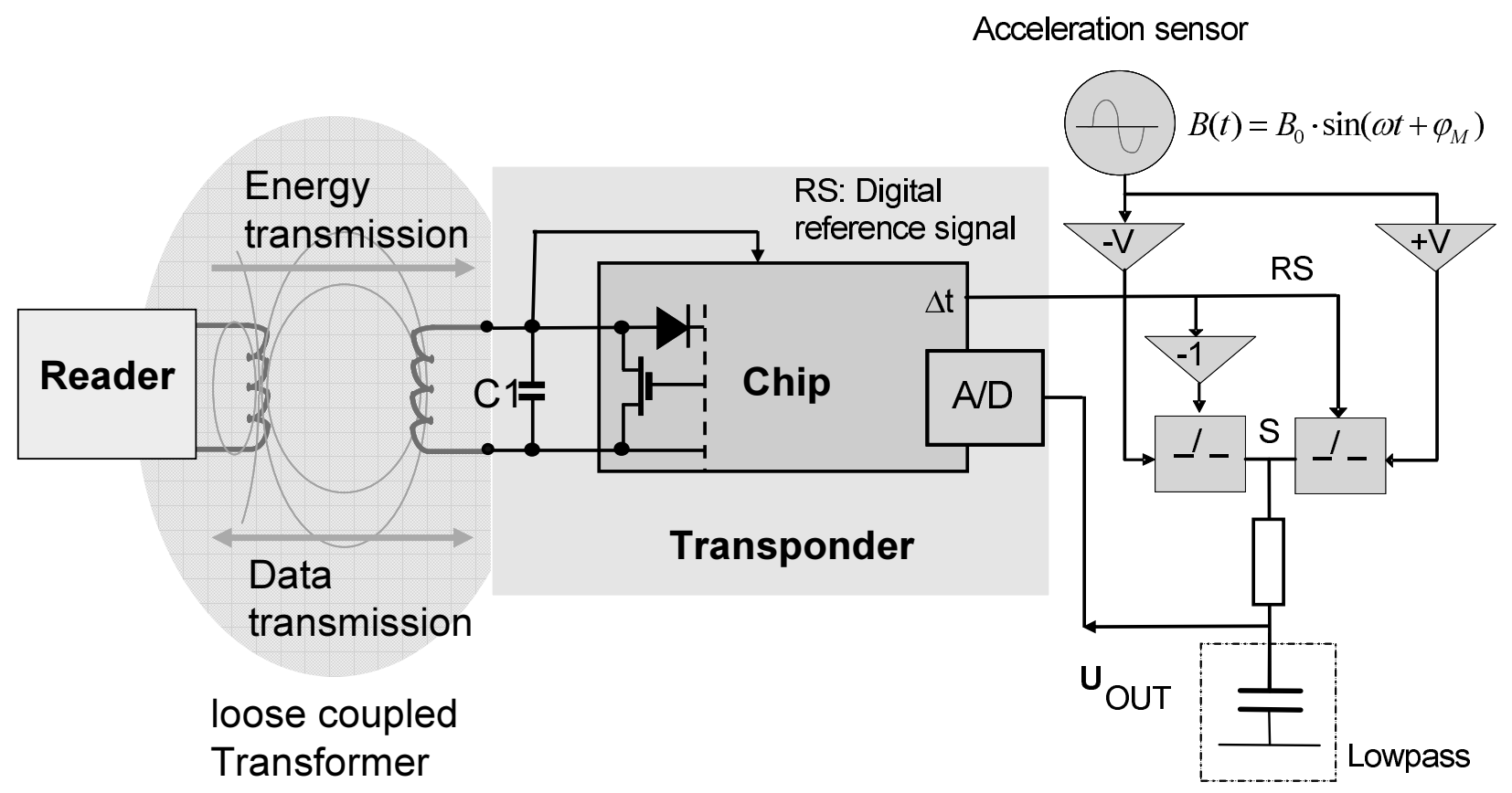

Figure 2: Transponder principle and integrated digital lock-in amplifier

\section{Prosthesis Vibration Excitation}

The resonance frequencies of the prosthesis were calculated by FEM simulations for different distal and proximal fixed supports of the prosthesis. The FEM simulation resulted in several resonances which are depicted up to $18 \mathrm{kHz}$ in Figure 3. The frequency values mainly depend on the region of distal fixation and the point forces acting. In experimental preliminary investigations miniaturized acceleration sensors 
had been integrated in hip prosthesis to determine their eigenfrequency pattern as indirect criterion for the state of a prosthesis loosening.

Further the shaker excitation of the prosthesis and the femur was developed and investigated. The goal was to excite a support plate inclusive sensor with its total mass as a whole sinusoidal by the electrodynamical shaker and to couple this movement to the femur above the knee. On the one hand for this the exciter mass of approx. $50-100 \mathrm{~g}$ must be moved with $10 \mathrm{~W}$ still between $800 \mathrm{~Hz}$ and $5 \mathrm{kHz}$, on the other hand we wish to include a sensor, which measures dynamically the force as reference for the lock-in procedure. The coupling to the human bones varies as well known. Natural oscillations, frequency doubling

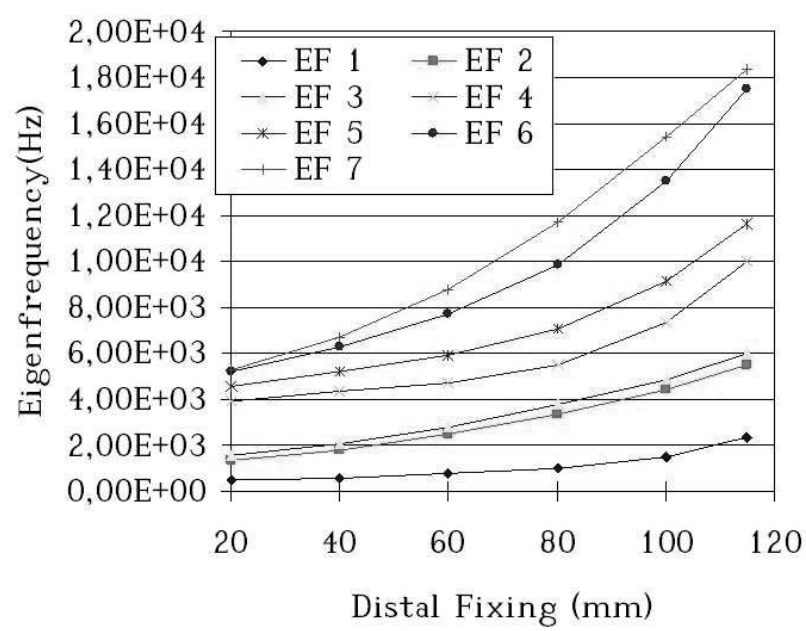

Figure 3: FEM simulated resonance frequencies of a proximal loose prosthesis

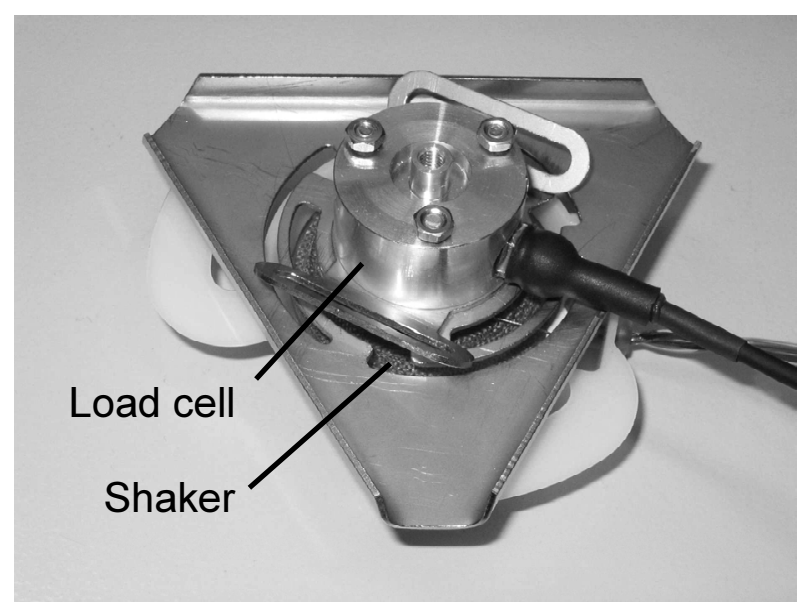

Figure 4: Shaker and load cell mounted at the support plate

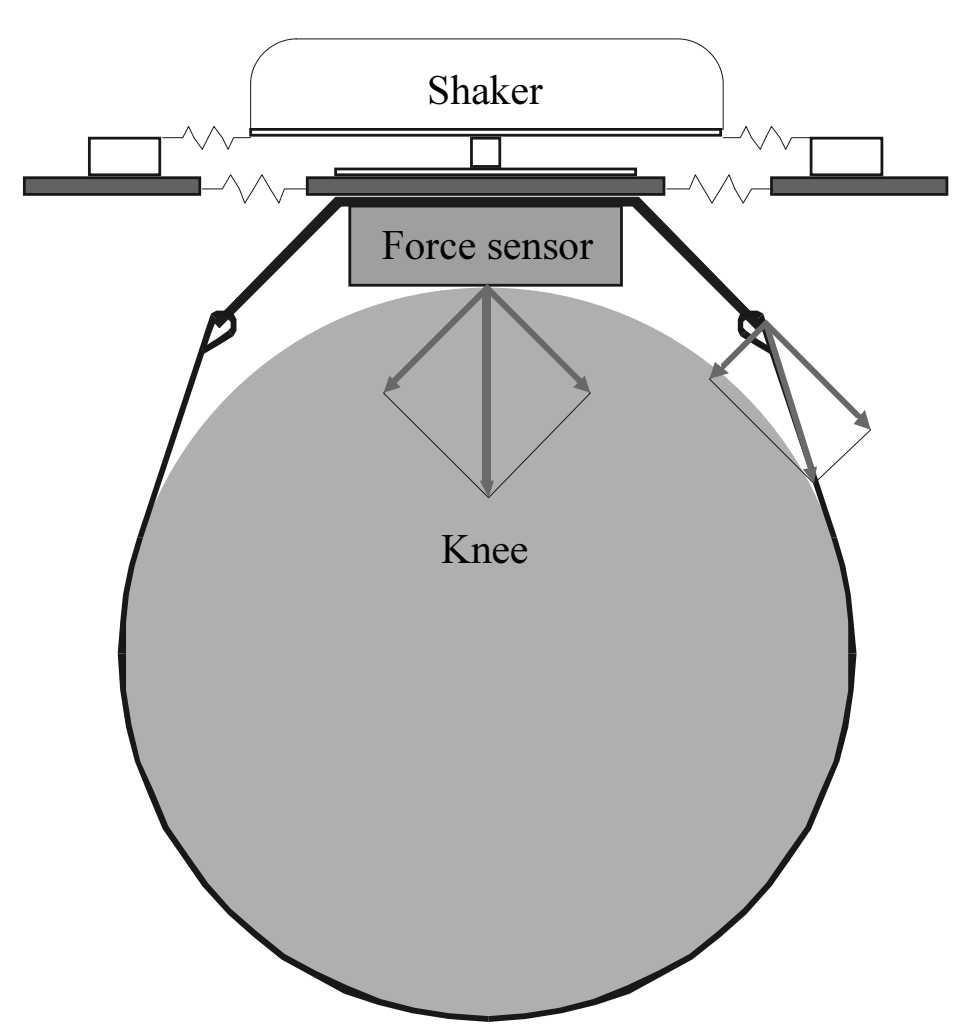

Figure 5: Application of the excitation force to the femur
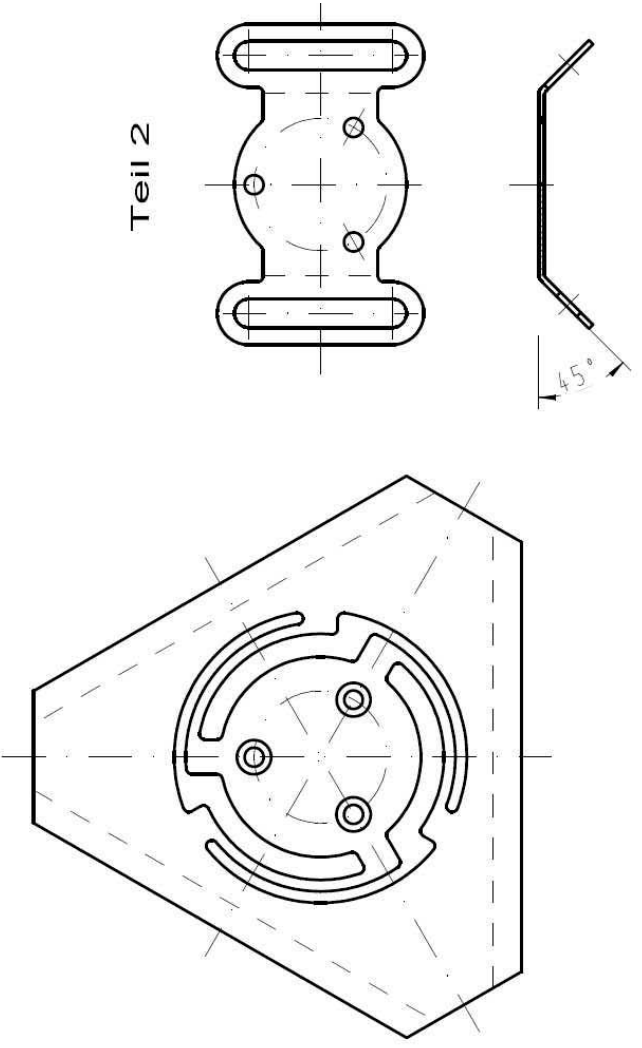

Figure 6: Design of the support plate 


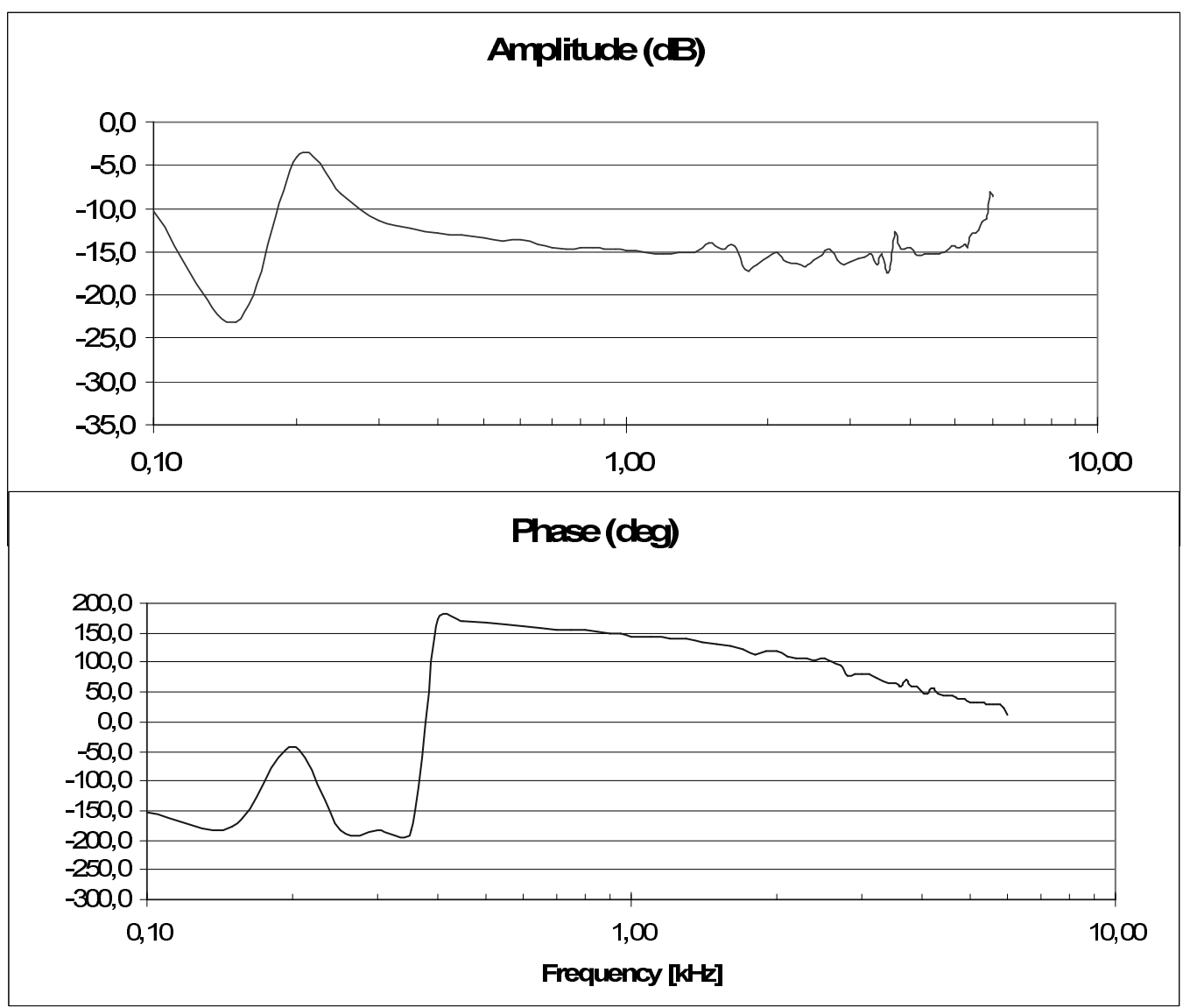

Figure 7: Amplitude and phase response of the exciter mounted on the support plate

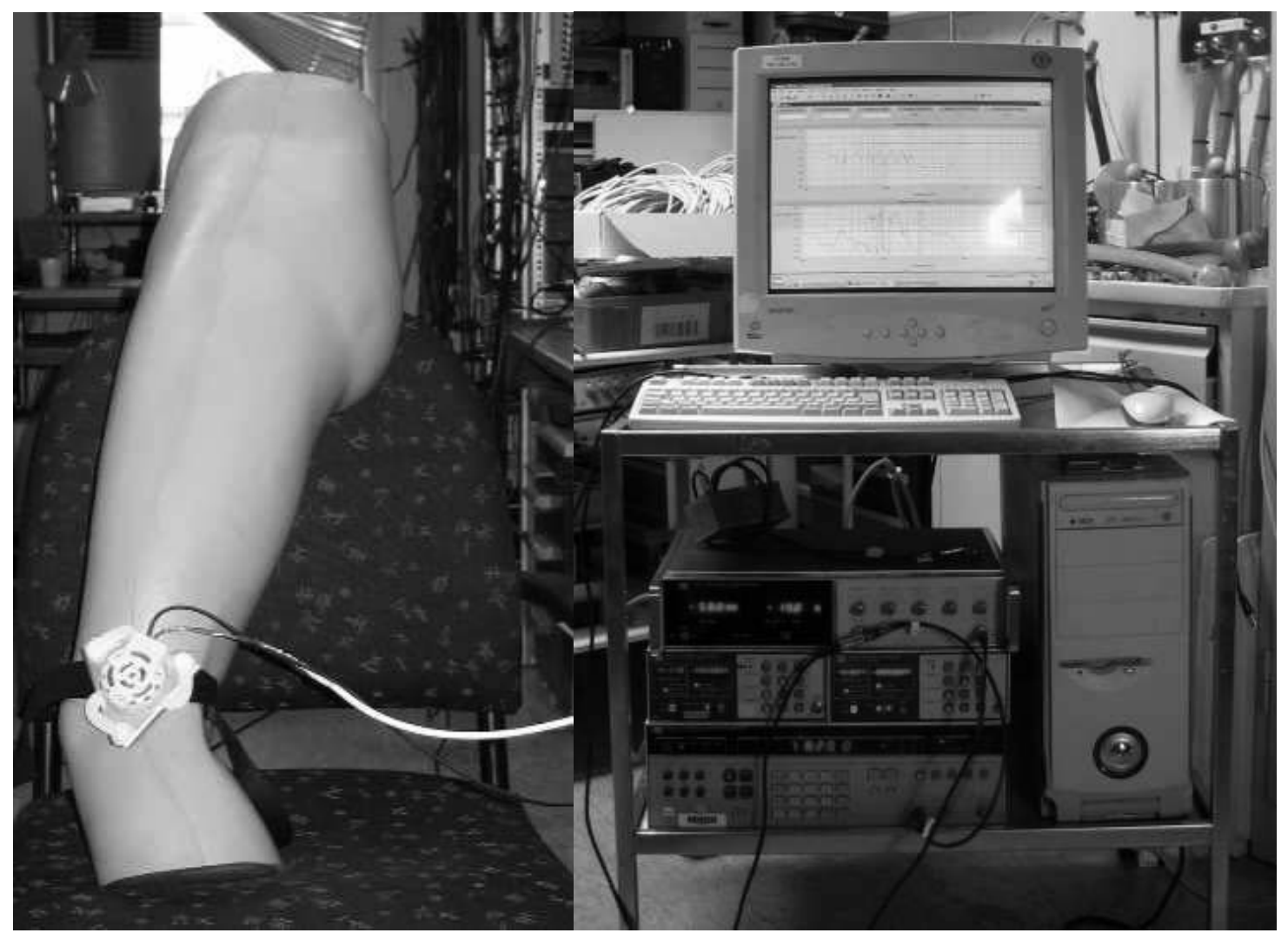

Figure 8: Laboratory setup at Berufsgenossenschaftliches Universitätsklinikum Bergmannsheil Bochum 
or phase shifts are unwanted thereby. Amplitudes of frequency portions above the generator frequency should drop steeply. In particular a $2 f$ suppression is necessary, since the lock-in is sensitive here. In particular the $1^{\text {st }}$ harmonic besides the fundamental oscillation of the prosthesis is needed to determine the strength of the anchorage.

As electrodynamical transducer (exciter/shaker) the RM02 audio vibration motor was chosen for cost reasons. It is characterized by a nominal impedance of $6 \mathrm{Ohm}$ at $1 \mathrm{kHz}$ and can be operated with a nominal input power of $5 \mathrm{~W}$. In order to apply the shaker vibrations at the knee the shaker was placed on a support plate which was fixed at the knee using a Velcroß strip. The strip was connected to two attachment handles which are depicted in Fig. 6 as Teil (part) 2. The necessary contact pressure was assured by an air cushion underneath the strip. Thus the anatomical form can be taken into account to a large extent above the knee joint. The air cushion is blown up conventionally with hand bellows up to a pressure of $60-80 \mathrm{~mm} \mathrm{Hg}$. The related contact force acting over a $10 \mathrm{~mm}$ aluminum head at the knee was measured with a A.S.T KAM-S load cell as shown in Figure 4 and 5.

Besides carrying the shaker the support plate separates its housing vibrations in the intersting frequency range from the force source by 3 bending spring structures. The amplitude and phase response is depicted in Fig. 7. In the frequency range above $300 \mathrm{~Hz}$ no resonances are present but the phase change has to be included in the lock-in amplifier synchronization especially for frequencies above $2.5 \mathrm{kHz}$.

\section{Measurements}

The measurement setup is shown in Figure 8 with the shaker fixed at the knee. The hip prosthesis was fixed distally in an artificial femur using cement which then was placed in an artificial thigh.

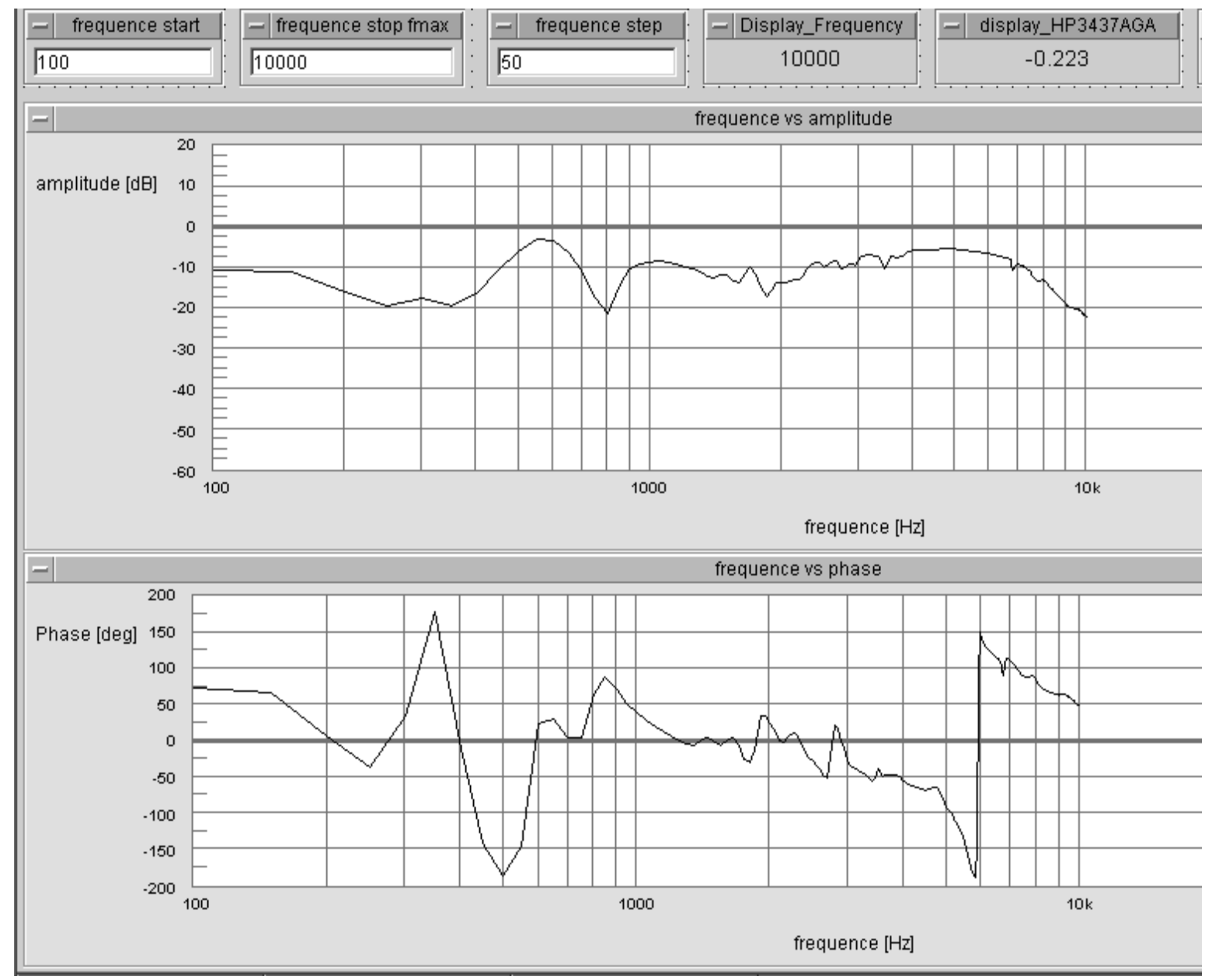

Figure 9: Frequency response measured with the acceleration sensor in the prosthesis, when the shaker was pressed on the knee with an air cushion where $80 \mathrm{mmHg}$ pressure were applied. The acceleration sensor measures the main vibrational plane which is excited by the shaker. 
According to the large phase shift the shaker resonance can be observed in Figure 9 between 400 and $500 \mathrm{~Hz}$ and above this frequency resonances of the bone-prosthesis compound. The phase shift at $6 \mathrm{kHz}$ and the amplitude decrease above are caused by a RC lowpass at the sensor output which serves as noise and antialiasing filter. The resonance frequency of the acceleration sensor is specified at $10 \mathrm{kHz}$. With adequate mechanical loosening models the fixed and the loose state can be distinguished based on the observable phase shifts between $500 \mathrm{~Hz}$ and $6 \mathrm{kHz}$.

\section{Conclusion}

An experimental setup for excitation and detection of hip prosthesis loosing was developed and analyzed. Applying an appropriate high shaker power the main vibrational plane can be excited at the distal end and measured telemetrically in the human body. Acceleration measurements are pre-processed with a lock-in amplifier in the prosthesis. An integrated controller and telemetry chip synchronizes the measurement, samples the lock-in output signal and transmits the data. This concept of a digital communication allows data storage in the prosthesis.

In the future besides patient and prosthesis manufacturer data also, for example, reference data sets could be stored which are needed for a loosening trend analysis. The lock-in amplification of the prosthesis vibration provides the amplitude of the oscillation, i.e. not a full set of measurements, sampled above Nyquist frequency, has to be transmitted. That saves time and energy.

\section{Acknowledgement}

This work was supported by the Bundesministerium für Wirtschaft und Technologie (BMWi), research project PROTEL, 16IN0287. The authors thank U. Holzwarth (Med-Titan, Nürnberg), E. Künzel (A.S.T. Dresden), S. Richter and M. Bertram (TU Dresden) for their support.

\section{References}

[1] D. Rosenstein et al: The differentiation of loose and secure femoral implants in total hip replacement using vibrational technique, an anatomical and pilot clinical study, Proc. Inst. Mech, Eng. 203 (1989) 77-81

[2] P.L. Li, N.B. Jones, and P.J. Gregg: Loosening of total hip arthroplasty. Diagnosis by vibration analysis, The Journal of Bone and Joint Surgery, Vol. 77-B, No. 4, July 1995

[3] S. Giardini, P. Cornwell, R.M. Meneghini: Monitoring femoral component installation using vibration testing. Biomed Sci Instrum. 41:13-8, 2005;

[4] G. Qi, W.P. Mouchon, T.E. Tan: How much can a vibrational diagnostic tool reveal in total hip arthroplasty loosening? Clin Biomech (Bristol, Avon). 2003 Jun;18(5):444-58.

[5] R. Puers et al: A telemetry system for the detection of hip prosthesis loosening by vibration analysis, Sensors and Actuators 85(2000) 42-47

[6] R. Puers et al: An Implantable System for Detecting Loosening of a Hip Prosthesis. In: Bio-telemtry 15, 2000, Ed. by Int.Soc. on Biotelemetry, Wageningen, The Netherlands, S. 652-660

[7] F. Burny, M. Donkerwolcke, F. Moulart, R. Bourgois, R. Puers, K. Van Schuylenbergh, M. Barbosad, O. Paivad, F. Rodes, J.B. Bégueret, P. Lawes: Concept, design and fabrication of smart orthopedic implants, Mechanical Engineering and Physics, Vol. 22 (7), pp. 469-479

[8] J. Parvizi, V. Antoci, Jr., N.J. Hickok, I.M. Shapiro: Selfprotective smart orthopedic implants. Expert. Rev. Med. Devices, 4:55-64, 2007

[9] Jettkant. B. et al: Design and Print of a Wireless Diagnostic For Detecting The Loosening of Protheses in Vitro Based on Acceleration Sensors. In: Proceedings of the World Congress on Medical Physics and Biomedical Engineering, Aug. 24-29, 2003, Sydney, Australia

[10] Jettkant. B. et al.: Implantierbare Telemetrien für Diagnostik, In: Biomedizinische Technik, Bd. 46, Ergänzungsband 1, S.394-395, 2001

[11] U. Marschner, H. Grätz, B. Jettkant, D. Ruwisch, G. Woldt, W.-J. Fischer and B. Clasbrummel: Integration of a Wireless Lock-In Measurement of Hip Prosthesis Vibrations for Loosening Detection, in Eurosensors XXII, Dresden, 7-10 September 2008 